\title{
LOWER SEMICONTINUITY FOR NONAUTONOMOUS SURFACE INTEGRALS
}

\author{
VIRGINIA DE CICCO
}

\begin{abstract}
Some lower semicontinuity results are established for nonautonomous surface integrals depending in a discontinuous way on the spatial variable. The proof of the semicontinuity results is based on some suitable approximations from below with appropriate functionals.
\end{abstract}

\section{INTRODUCTION}

In this paper new lower semicontinuity results are obtained for nonautonomous surface integrals whose dependence on the spatial variable is discontinuous. Surface energies of this type occur in free discontinuity problems, as in fracture mechanics when one considers quasistatic evolution of stratified, heterogeneous materials (see for instance [17], [18], [20] and [21]).

The surface energy usually admits the form

$$
\Phi(u):=\int_{\Omega \cap J_{u}} \phi\left(u^{-}(x), u^{+}(x), \nu_{u}(x)\right) d \mathcal{H}^{N-1}, \quad u \in S B V\left(\Omega ; \mathbb{R}^{m}\right),
$$

where $\Omega$ is an open bounded subset of $\mathbb{R}^{N}, S B V\left(\Omega ; \mathbb{R}^{m}\right)$ is the space of the vector valued special function of bounded variation, $\mathcal{H}^{N-1}$ denotes the $(N-1)$-dimensional Hausdorff measure and $\phi$ is a jointly convex function, depending on the traces of $u$ on the jump set $J_{u}$ and its orientation $\nu_{u}$ (see [10] and [11]). The proof of the lower semicontinuity of the surface integral $\Phi$ is obtained by considering some approximating functionals constructed by using the definition of jointly convex function (see Def. 2.8) and by using for the approximating functionals the chain rule formula for vector valued functions in $B V$ (see Theorem 5.22 in [13]).

2010 Mathematics Subject Classification. 49J45, 49Q20.

Key words and phrases. Semicontinuity, capacity, chain rule, $B V$ functions. 
However, in some context the energy can admit an explicit dependence on the spatial variable $x$ and the following form

$$
\Phi(u):=\int_{\Omega \cap J_{u}} \phi\left(x, u^{-}(x), u^{+}(x), \nu_{u}(x)\right) d \mathcal{H}^{N-1} .
$$

When this dependence is discontinuous, these functionals permit to describe the case of heterogeneous and anisotropic materials (see [30]).

In the paper [5] it is considered a nonautonomous surface energy of the type

$$
\Phi(u):=\int_{\Omega \cap J_{u}} a(x) \varphi\left(u^{-}(x), u^{+}(x), \nu_{u}(x)\right) d \mathcal{H}^{N-1},
$$

where $\varphi$ is jointly convex and $a$ is a $W^{1,1}$ function. Moreover, in the same paper it is considered a surface energy of the type

$$
\Phi(u):=\int_{\Omega \cap J_{u}} \gamma\left(\left|u^{+}(x)-u^{-}(x)\right|\right) \varphi\left(x, \nu_{u}(x)\right) d \mathcal{H}^{N-1},
$$

where $\left|u^{+}-u^{-}\right|$is the difference of the trace of $u$ on both sides of $J_{u}, \nu_{u}$ is the normal to the jump set $J_{u}$ and the function $\gamma$ depends on the material. The integrand $\phi(x, r, t, \xi)=\gamma(|r-t|) \varphi(x, \xi)$ is an example of jointly convex integrand in $(r, t, \xi)$ (see Remark 3.3 for the assumptions on $\gamma$ and $\varphi$ ). For $\varphi(x, \xi)=1$ the energy was proposed by Barenblatt in [14], while in [18], [20] and [30] the authors consider the case where $\gamma(s)=1$ for every $s>0$ and $\gamma(0)=0$. For the function $\varphi(\cdot, \xi)$ it is required a $B V$ dependence on $x$.

The purpose of this paper is to study the lower semicontinuity of (1.2) for general nonautonomous jointly convex integrands. We will prove a lower semicontinuity theorem for the functional (1.2), along sequences $\left\{u_{n}\right\}$ in $\operatorname{SBV}^{p}\left(\Omega ; \mathbb{R}^{m}\right)(p>1)$ such that $u_{n}(x) \rightarrow u(x)$ for almost every $x \in \Omega$ and $\left\|\nabla u_{n}\right\|_{p}, \mathcal{H}^{N-1}\left(J_{u_{n}}\right)$ are uniformly bounded with respect to $n \in \mathbb{I N}$.

The interest of the results presented here is that the function $\phi$ may possibly be discontinuous with respect to $x$ and it admits a general structure with respect to the jointly convex integrands considered in [5]. The structural assumptions on $\phi(x, r, t, \xi)$ are a $W^{1,1}$ or $B V$ dependence on $x$ and the joint convexity in the last three variables; moreover some additional uniformity assumptions are required. 
In order to prove the lower semicontinuity of the surface integral some methods introduced previously in [5] are used here (see also [1], [2], [3], [4], [22], [23] and [24]).

In Section 3 and 4 we present two independent approaches, by giving different definitions of non autonomous jointly convex integrand and by using several approximation techniques from below.

In Section 3 the lower semicontinuity result is obtained via the nonautonomous chain rule formula (for vector valued $B V$ functions, recently proven in [12], or for scalar $B V$ function, proven in [22]). An explicit approximation of convex functions due to De Giorgi (see [25]), here adapted to jointly convex functions, allows to verify the regularity assumptions and the uniformity conditions of the approximating integrand and hence to apply the chain rule (see Prop. 3.3 below).

In Section 4, we study a very general case of $B V$ or $W^{1,1}$ dependence on $x$. Here the lower semicontinuity is obtained by approximating the integrand from below by jointly convex functions lower semicontinuous in $x$ uniformly with respect to the other variables. In this context we need to require a strict positivity assumption of the integrand.

\section{NOTATION AND PRELIMINARIES}

2.1. Notation. Throughout the paper $N>1, m \geq 1$ are fixed integers. Let $\Omega$ be a bounded open subset of $\mathbb{R}^{N}$ with Lipschitz boundary. We denote by $\mathbb{S}^{N-1}$ the unit sphere in $\mathbb{R}^{N}$. Let $\mathcal{L}^{N}$ denote the Lebesgue measure on $\mathbb{R}^{N}$ and $\mathcal{H}^{N-1}$ the Hausdorff measure of dimension $N-1$ on $\mathbb{R}^{N}$.

If $u \in L_{\mathrm{loc}}^{1}\left(\Omega ; \mathbb{R}^{m}\right)$ and $x \in \Omega$, the precise representative of $u$ at $x$ is defined as the unique value $\widetilde{u}(x) \in \mathbb{R}^{m}$ such that

$$
\lim _{\rho \rightarrow 0^{+}} \frac{1}{\rho^{N}} \int_{B_{\rho}(x)}|u(y)-\widetilde{u}(x)| d y=0 .
$$

In this case $u$ is said approximate continuous at $x$ and $\widetilde{u}(x)$ is the so-called approximate limit of $u$ at $x$. The set of points in $\Omega$ where the precise representative of $x$ is not defined is called the approximate singular set of $u$ and denoted by $S_{u}$. 
Let $u \in L_{\text {loc }}^{1}\left(\Omega ; \mathbb{R}^{m}\right)$ and $x \in \Omega$. We say that $x$ is an approximate jump point of $u$ if there exist $a, b \in \mathbb{R}^{m}$ and $\nu \in \mathbb{S}^{N-1}$, such that $a \neq b$ and

$$
\lim _{\rho \rightarrow 0^{+}} f_{B_{\rho}^{+}(x, \nu)}|u(y)-a| d y=0 \quad \text { and } \quad \lim _{\rho \rightarrow 0^{+}} f_{B_{\rho}^{-}(x, \nu)}|u(y)-b| d y=0
$$

where $B_{\rho}^{ \pm}(x, \nu):=\left\{y \in B_{\rho}(x):\langle y-x, \nu\rangle \gtrless 0\right\}$. The triplet $(a, b, \nu)$ is uniquely determined by the previous formulas, up to a permutation of $a, b$ and a change of sign of $\nu$, and it is denoted by $\left(u^{+}(x), u^{-}(x), \nu_{u}(x)\right)$. The Borel functions $u^{+}$and $u^{-}$are called the upper and lower approximate limit of $u$ at the point $x \in \Omega$. The set of approximate jump points of $u$ is defined by

$$
J_{u}=\left\{x \in \Omega: u^{+}(x) \neq u^{-}(x)\right\} .
$$

We recall that the space $\operatorname{BV}\left(\Omega ; \mathbb{R}^{m}\right)$ of functions of bounded variation is defined as the set of all $u \in L^{1}\left(\Omega ; \mathbb{R}^{m}\right)$ whose distributional gradient $D u$ is a bounded Radon measure on $\Omega$ with values in the space $M^{m \times N}$ of $m \times N$ matrices.

We recall the usual decomposition

$$
D u=\nabla u \mathcal{L}^{N}+D^{c} u+\left(u^{+}-u^{-}\right) \otimes \nu_{u} \mathcal{H}^{N-1}\left\lfloor J_{u},\right.
$$

where $\nabla u$ is the Radon-Nikodým derivative of $D u$ with respect to the Lebesgue measure and $D^{c} u$ is the Cantor part of $D u$. For the sake of simplicity, we denote by $D^{s} u=D^{c} u+\left(u^{+}-u^{-}\right) \otimes \nu_{u} \mathcal{H}^{N-1}\left\lfloor J_{u}\right.$.

We recall that the space $\operatorname{SBV}\left(\Omega ; \mathbb{R}^{m}\right)$ of special functions of bounded variation is defined as the set of all $u \in \operatorname{BV}\left(\Omega ; \mathbb{R}^{m}\right)$ such that $D^{s} u$ is concentrated on $S_{u}$; i.e., $\left|D^{s} u\right|\left(\Omega \backslash S_{u}\right)=0$.

Let $p>1$. The space $\operatorname{SBV}^{p}\left(\Omega ; \mathbb{R}^{m}\right)$ is the set of functions $u \in \operatorname{SBV}\left(\Omega ; \mathbb{R}^{m}\right)$ with $\nabla u \in L^{p}\left(\Omega ; M I^{m \times N}\right)$ and $\mathcal{H}^{N-1}\left(S_{u}\right)<\infty$. A sequence $\left\{u_{n}\right\}$ converges to $u$ weakly in $\operatorname{SBV}^{p}\left(\Omega ; \mathbb{R}^{m}\right) \cap L^{\infty}\left(\Omega ; \mathbb{R}^{m}\right)$ if $u_{n}(x) \rightarrow u(x)$ almost everywhere in $\Omega, \nabla u_{n} \rightarrow \nabla u$ weakly in $L^{p}\left(\Omega ; M^{m \times N}\right)$, and $\left\|u_{n}\right\|_{\infty}$ and $\mathcal{H}^{N-1}\left(S_{u_{n}}\right)$ are bounded uniformly with respect to $n$.

For a general survey on the spaces of $\mathrm{BV}, \mathrm{SBV}$ and $\mathrm{SBV}^{p}$ functions we refer to [13].

2.2. Approximation results. Now we recall some approximation results that will be used in the sequel. In the next lemma it is obtained the lower semicontinuity for a functional whose integrand is the supremum of convex functions (see [29]). 
Lemma 2.1. Let $h, h_{j}: \Omega \times \mathbb{R}^{m} \times \mathbb{R}^{m} \times \mathbb{R}^{N} \rightarrow[0,+\infty), j \in \mathbb{N}$, be Borel functions, convex and positively 1-homogeneous in the last variable and such that

$$
h(x, r, t, \xi)=\sup _{j \in \mathbb{N}} h_{j}(x, r, t, \xi)
$$

for all $(x, r, t, \xi) \in\left(\Omega \backslash N_{0}\right) \times \mathbb{R}^{m} \times \mathbb{R}^{m} \times \mathbb{R}^{N}$, where $N_{0} \subset \Omega$ is a Borel set with $\mathcal{H}^{N-1}\left(N_{0}\right)=0$. If the functionals $\mathcal{F}_{h_{j}}$ defined by

$$
\mathcal{F}_{h_{j}}(u):=\int_{\Omega \cap J_{u}} h_{j}\left(x, u^{-}, u^{+}, \nu_{u}\right) d \mathcal{H}^{N-1}
$$

are weakly lower semicontinuous in $\mathrm{SBV}^{p}\left(\Omega ; \mathbb{R}^{m}\right)$, then $\mathcal{F}_{h}$, defined similarly, is weakly lower semicontinuous in $\mathrm{SBV}^{p}\left(\Omega ; \mathbb{R}^{m}\right)$ too.

The following lemma is a classical approximation result due to De Giorgi (see [25] and also Thm. 4.79 in [27]).

Lemma 2.2. There exists a sequence $\left\{\alpha_{k}\right\} \subset C_{c}^{\infty}\left(\mathbb{R}^{N}\right)$, with $\alpha_{k} \geq 0$ and $\int_{\mathbb{R}^{N}} \alpha_{k}(\xi) d \xi=1$ such that, if $f: \Omega \times \mathbb{R}^{N} \rightarrow[0,+\infty)$ is a function convex in the last variable and we consider

$$
\begin{aligned}
& a_{0, k}(x)=\int_{\mathbb{R}^{N}} f(x, \xi)\left((N+1) \alpha_{k}(\xi)+\left\langle\nabla \alpha_{k}(\xi), \xi\right\rangle\right) d \xi \\
& a_{i, k}(x)=-\int_{\mathbb{R}^{N}} f(x, \xi) \frac{\partial}{\partial \xi_{i}} \alpha_{k}(\xi) d \xi, \quad i=1, \cdots, N
\end{aligned}
$$

and $a_{k}=\left(a_{1, k}, \ldots, a_{N, k}\right)$, then for all $(x, r, t, \xi) \in \Omega \times \mathbb{R}^{N}$ we have

$$
f(x, \xi)=\sup _{k \in \mathbb{N}}\left[a_{0, k}(x)+\left\langle a_{k}(x), \xi\right\rangle\right]^{+} .
$$

If $f$ is also positively 1-homogeneous, then

$$
f(x, \xi)=\sup _{k \in \mathbb{N}}\left\langle a_{k}(x), \xi\right\rangle^{+} .
$$

2.3. Capacity. Given an open set $A \subset \mathbb{R}^{N}$, the 1-capacity of $A$ is defined by setting

$$
C_{1}(A):=\inf \left\{\int_{\mathbb{R}^{N}}|D \varphi| d x: \varphi \in W^{1,1}\left(\mathbb{R}^{N}\right), \quad \varphi \geq 1 \quad \mathcal{L}^{N} \text {-a.e. on } A\right\} .
$$


Then, the 1-capacity of an arbitrary set $B \subset \mathbb{R}^{N}$ is given by

$$
C_{1}(B):=\inf \left\{C_{1}(A): A \supseteq B, A \text { open }\right\} .
$$

It is well known that for every Borel set $B \subset \mathbb{R}^{N}$

$$
C_{1}(B)=0 \quad \Longleftrightarrow \quad \mathcal{H}^{N-1}(B)=0 .
$$

Definition 2.3. Let $B \subset \mathbb{R}^{N}$ be a Borel set with $C_{1}(B)<+\infty$. Given $\varepsilon>0$, we call capacitary $\varepsilon$-quasi-potential (or simply capacitary quasipotential) of $B$ a function $\varphi_{\varepsilon} \in W^{1,1}\left(\mathbb{R}^{N}\right)$, such that $0 \leq \widetilde{\varphi}_{\varepsilon} \leq 1 \mathcal{H}^{N-1}$-a.e. in $\mathbb{R}^{N}, \widetilde{\varphi}_{\varepsilon}=1 \mathcal{H}^{N-1}$-a.e. in $B$ and

$$
\int_{\mathbb{R}^{N}}\left|D \varphi_{\varepsilon}\right| d x \leq C_{1}(B)+\varepsilon .
$$

We recall that a function $g: \mathbb{R}^{N} \rightarrow \mathbb{R}$ is said $C_{1}$-quasi continuous if for every $\varepsilon>0$ there exists an open set $A$, with $C_{1}(A)<\varepsilon$, such that $\left.g\right|_{A^{c}}$ is continuous on $A^{c} ; C_{1}$-quasi lower semicontinuous and $C_{1}$-quasi upper semicontinuous functions are defined similarly.

It is well known that if $g$ is a $W^{1,1}$ function, then its precise representative $\widetilde{g}$ is $C_{1}$-quasi continuous (see [26, Sections 9 and 10]). Moreover, to every BV function $g$, it is possible to associate a $C_{1}$-quasi lower semicontinuous and a $C_{1}$-quasi upper semicontinuous representative, as stated by the following theorem (see [15], Theorem 2.5).

Theorem 2.4. For every function $g \in \mathrm{BV}(\Omega)$, the approximate upper limit $g^{+}$and the approximate lower limit $g^{-}$are $C_{1}$-quasi upper semicontinuous and $C_{1}$-quasi lower semicontinuous, respectively.

Moreover we recall the following lemma which is an approximation result due to Dal Maso (see [16], Lemma 1.5 and $\S 6$ ).

Lemma 2.5. Let $g: \mathbb{R}^{N} \rightarrow[0,+\infty)$ be a $C_{1}$-quasi lower semicontinuous function. Then there exists an increasing sequence of nonnegative functions $\left\{g_{h}\right\} \subseteq W^{1,1}\left(\mathbb{R}^{N}\right)$ such that, for every $h \in \mathbb{N}, g_{h}$ is approximately continuous $\mathcal{H}^{N-1}$-almost everywhere in $\mathbb{R}^{N}$ and $g_{h}(x) \rightarrow g(x)$, when $h \rightarrow+\infty$, for $\mathcal{H}^{N-1}$-almost every $x \in \mathbb{R}^{N}$. 


\subsection{Chain rules.}

2.4.1. Vectorial case. We recall a chain rule formula in $S B V$ which is a particular case of a chain rule in $B V$ recently obtained in [12] under more general assumptions on the dependence in $x$.

Let $g: \mathbb{R}^{N} \times \mathbb{R}^{m} \rightarrow \mathbb{R}^{N}$ be satisfying:

(a) $x \mapsto g(x, r)$ belongs to $W_{\text {loc }}^{1,1}\left(\mathbb{R}^{N} ; \mathbb{R}^{N}\right)$ for all $r \in \mathbb{R}^{m}$;

(b) there exist a positive function $h \in L_{\mathrm{loc}}^{1}\left(\mathbb{R}^{N}\right)$ and a modulus of continuity $\omega$ such that

$$
\left|\nabla_{x} g(x, r)-\nabla_{x} g\left(x, r^{\prime}\right)\right| \leq \omega\left(\left|r-r^{\prime}\right|\right) h(x)
$$

for all $r, r^{\prime} \in \mathbb{R}^{m}$ and for $\mathcal{L}^{N}$-a.e. $x \in \mathbb{R}^{N}$;

(c) there exists a Lebesgue negligible set $N \subset \mathbb{R}^{N}$ such that $r \mapsto g(x, r)$ is continuously differentiable in $\mathbb{R}^{m}$ for all $x \in \mathbb{R}^{N} \backslash N$;

(d) for some constant $M,\left|\nabla_{r} g(x, r)\right| \leq M$ for all $x \in \mathbb{R}^{N} \backslash N$ and $r \in \mathbb{R}^{m}$;

(e) for any compact set $H \subset \mathbb{R}^{m}$ there exists a modulus of continuity $\widetilde{\omega}_{H}$ independent of $x$ such that

$$
\left|\nabla_{r} g(x, r)-\nabla_{r} g\left(x, r^{\prime}\right)\right| \leq \widetilde{\omega}_{H}\left(\left|r-r^{\prime}\right|\right)
$$

for all $r, r^{\prime} \in H$ and $x \in \mathbb{R}^{N} \backslash N$.

Theorem 2.6. Let $g$ be satisfying (a), (b), (c), (d) and (e) above. Then there exists a set $\mathcal{N} \subset \mathbb{R}^{N}$ with $\mathcal{H}^{N-1}(\mathcal{N})=0$, such that, for any function $u \in S B V_{\text {loc }}\left(\mathbb{R}^{N} ; \mathbb{R}^{m}\right)$, the function $v(x):=g(x, u(x))$ belongs to $S B V_{\text {loc }}\left(\mathbb{R}^{N} ; \mathbb{R}^{N}\right)$ and the following chain rule holds:

(i) for every $r \in \mathbb{R}^{m}$ the function $g(\cdot, r)$ is approximately continuous in $\mathbb{R}^{N} \backslash \mathcal{N}$ and $\widetilde{g}(x, r)$ denotes the precise representative of $g(\cdot, r)$ on $\mathbb{R}^{N} \backslash \mathcal{N}$

(ii) (Lebesgue part) for $\mathcal{L}^{N}$-a.e. $x$ the map $y \mapsto g(y, u(x))$ is approximately differentiable at $x$ and

$$
\nabla v(x)=\left(\nabla_{x} g\right)(x, u)+\left(\nabla_{r} g\right)(x, u) \cdot \nabla u(x) \quad \mathcal{L}^{N} \text {-a.e. in } \mathbb{R}^{N} ;
$$

(iii) (jump part) $J_{v} \subset J_{u}$ and it holds

$$
D^{j} v=\left(\widetilde{g}\left(x, u^{+}\right)-\widetilde{g}\left(x, u^{-}\right) \otimes \nu_{u} \mathcal{H}^{N-1}\left\llcorner J_{u}\right.\right.
$$

in the sense of measures, where $u^{ \pm}(x)$ are the upper and lower approximate limits of $u$ at $x$. 
Moreover

$$
\begin{aligned}
\operatorname{div} v(x)= & {\left[\left(\operatorname{div}_{x} g\right)(x, u)+\operatorname{tr}\left(\left(\nabla_{r} g\right)(x, u) \nabla u\right)\right] \mathcal{L}^{N} } \\
& +\left\langle\widetilde{g}\left(x, u^{+}\right)-\widetilde{g}\left(x, u^{-}\right), \nu_{u}\right\rangle \mathcal{H}^{N-1}\left\llcorner J_{u}\right.
\end{aligned}
$$

in the sense of measures.

2.4.2. Scalar case. We recall a chain rule formula for scalar functions proven in $[22]$.

Let $g: \mathbb{R}^{N} \times \mathbb{R} \rightarrow \mathbb{R}^{N}$ be satisfying:

(A) the function $x \mapsto g(x, r)$ belongs to $W^{1,1}\left(\mathbb{R}^{N} ; \mathbb{R}^{N}\right)$ for all $r \in \mathbb{R}$ and there exists a positive constant $M$ such that for all $r \in \mathbb{R}$

$$
\int_{\mathbb{R}^{N}}\left|\nabla_{x} g(x, r)\right| d x \leq M ;
$$

(B) there exists a Lebesgue negligible set $N \subset \mathbb{R}^{N}$ such that $r \mapsto g(x, r)$ is Lipschitz continuous in $\mathbb{R}$ for all $x \in \mathbb{R}^{N} \backslash N$;

(C) for some constant $M,\left|\nabla_{r} g(x, r)\right| \leq M$ for all $x \in \mathbb{R}^{N} \backslash N$ and $r \in \mathbb{R}$.

Theorem 2.7. Let $g$ be satisfying (A), (B) and $(\mathrm{C})$ above. Then there exists a set $\mathcal{N} \subset \mathbb{R}^{N}$ with $\mathcal{H}^{N-1}(\mathcal{N})=0$, such that, for any function $u \in$ $S B V_{\mathrm{loc}}\left(\mathbb{R}^{N}\right)$, the function $v(x):=g(x, u(x))$ belongs to $S B V_{\mathrm{loc}}\left(\mathbb{R}^{N} ; \mathbb{R}^{N}\right)$ and the following chain rule holds:

(I) for every $r \in \mathbb{R}$ the function $g(\cdot, r)$ is approximately continuous in $\mathbb{R}^{N} \backslash \mathcal{N}$ and $\widetilde{g}(x, r)$ denotes the precise representative of $g(\cdot, r)$ on $\mathbb{R}^{N} \backslash \mathcal{N}$

(II) (Lebesgue part) for $\mathcal{L}^{N}$-a.e. $x$ the map $y \mapsto g(y, u(x))$ is approximately differentiable at $x$ and

$$
\nabla v(x)=\left(\nabla_{x} g\right)(x, u)+\left(\nabla_{r} g\right)(x, u) \cdot \nabla u \quad \mathcal{L}^{N} \text {-a.e. in } \mathbb{R}^{N} ;
$$

(III) (jump part) $J_{v} \subset J_{u}$ and it holds

$$
D^{j} v=\left(\widetilde{g}\left(x, u^{+}\right)-\widetilde{g}\left(x, u^{-}\right) \cdot \nu_{u} \mathcal{H}^{N-1}\left\llcorner J_{u}\right.\right.
$$

in the sense of measures, where $u^{ \pm}$are the upper and lower approximate limits of $u$ at $x$.

Moreover

$$
\begin{aligned}
\operatorname{div} v(x) & =\left[\left(\operatorname{div}_{x} g\right)(x, u)+\left(\nabla_{r} g\right)(x, u) \nabla u\right] \mathcal{L}^{N} \\
& +\left\langle\widetilde{g}\left(x, u^{+}\right)-\widetilde{g}\left(x, u^{-}\right), \nu_{u}\right\rangle \mathcal{H}^{N-1}\left\llcorner J_{u}\right.
\end{aligned}
$$


in the sense of measures.

\subsection{Jointly convex functions.}

Definition 2.8. Let $K \subset \mathbb{R}^{m}$ be a compact set and $\phi: K \times K \times \mathbb{R}^{N} \rightarrow$ $[0,+\infty)$. We say that $\phi$ is jointly convex if there exists a sequence of functions $g_{j} \in \mathcal{C}\left(K ; \mathbb{R}^{N}\right)$ such that

$$
\phi(r, t, \xi)=\sup _{j \in \mathbb{N}}\left\langle g_{j}(r)-g_{j}(t), \xi\right\rangle \quad \text { for all }(r, t, \xi) \in K \times K \times \mathbb{R}^{N} .
$$

We remark that if $\phi$ is jointly convex, then

$\left(J_{1}\right) \phi(r, r, \xi)=0$;

$\left(J_{2}\right)$ (subadditivity) $\phi(r, t, \xi) \leq \phi(r, s, \xi)+\phi(s, t, \xi)$ for all $r, s, t \in K$ and $\xi \in \mathbb{R}^{N}$

$\left(J_{3}\right)$ (simmetry) $\phi(r, t, \xi)=\phi(t, r,-\xi)$ for all $r, t \in K$ and $\xi \in \mathbb{R}^{N}$;

$\left(J_{4}\right) \phi$ is convex, positively 1-homogeneous in $\xi$.

Remark 2.9. As in Example 5.23 in [13] some classes of jointly convex functions $\phi$ can be obtained in the following way:

(E1) Let $\phi: K \times K \times \mathbb{R}^{N} \rightarrow[0,+\infty)$

$$
\phi(r, t, \xi)=\gamma(|r-t|) \varphi(\xi)
$$

where $\gamma$ is a lower semicontinuous, increasing and subadditive function with $\gamma(0)=0$ and $\varphi$ is lower semicontinuous, convex, positively 1-homogeneous and even.

(E2) Let $\phi: K \times K \times \mathbb{R}^{N} \rightarrow[0,+\infty)$

$$
\phi(r, t, \xi)=\vartheta(r, t) \varphi(\xi),
$$

where $\vartheta: K \times K \rightarrow[0,+\infty)$ is a continuous function and it is a pseudodistance in $K$ (i.e. a positive, symmetric function satisfying the triangle inequality) and $\varphi: \mathbb{R}^{N} \rightarrow[0,+\infty]$ is lower semicontinuous, convex, positively 1-homogeneous and even.

\section{Nonautonomous Jointly Convex functions}

We give a definition of nonautonomous (NA) jointly convex function with $W^{1,1}$ dependence of the approximating functions with respect to the spatial variable $x$. 
Definition 3.1. Let $K \subset \mathbb{R}^{m}$ be a compact set and $\phi: \Omega \times K \times K \times \mathbb{R}^{N} \rightarrow$ $[0,+\infty)$. We say that $\phi$ is $N A$ jointly convex if there exists a sequence of functions $g_{j}: \Omega \times K \rightarrow \mathbb{R}^{N}$ such that

$\phi(x, r, t, \xi)=\sup _{j \in \mathbb{N}}\left\langle g_{j}(x, r)-g_{j}(x, t), \xi\right\rangle \quad$ for all $(x, r, t, \xi) \in \Omega \times K \times K \times \mathbb{R}^{N}$ and for every $j \in \mathbb{N}$ the function $g_{j}$ satisfies conditions (a), (b), (c), (d) and (e) of the vectorial chain rule or, if $m=1$, the function $g_{j}$ satisfies conditions (A), (B) and (C) of the scalar chain rule.

Remark 3.2. We give some example of NA jointly convex functions. The model case is

$$
\phi(x, r, t, \xi):=\langle g(x, r)-g(x, t), \xi\rangle^{+},
$$

where $g$ satisfies conditions (a), (b), (c), (d) and (e) of the vectorial chain rule (or (A), (B) and (C) of the scalar chain rule). A further example is

$$
\phi(x, r, t, \xi):=a(x) \varphi(r, t, \xi),
$$

where $a$ is a nonnegative bounded $W^{1,1}$ function,

$$
\varphi(r, t, \xi)=\sup _{j \in \mathbb{N}}\left\langle h_{j}(r)-h_{j}(t), \xi\right\rangle^{+}
$$

and $h_{j}$ are $C^{1}$ functions with bounded derivatives.

Another example of a NA jointly convex function is given in the following proposition.

Proposition 3.3. Let $\phi: \Omega \times K \times K \times \mathbb{R}^{N} \rightarrow[0,+\infty)$ be a locally bounded function such that

$$
\phi(x, r, t, \xi):=\vartheta(r, t) \varphi(x, \xi),
$$

where

$\left(i_{1}\right) \vartheta$ is a continuous function and it is a pseudo-distance in $K$ (i.e. a positive, symmetric function satisfying the triangle inequality);

$\left(i_{2}\right) \varphi$ is convex, positively 1-homogeneous and even in $\xi$ and there exists a constant $L>0$ such that

$$
\left|\phi(x, \xi)-\phi\left(x, \xi^{\prime}\right) \leq L\right| \xi-\xi^{\prime} \mid \quad \forall x \in \Omega \quad \forall \xi, \xi^{\prime} \in \mathbb{R}^{N} ;
$$

$\left(i_{3}\right)$ for every $\xi \in \mathbb{R}^{N}$ the map $x \mapsto \varphi(x, \xi)$ belongs to $W^{1,1}(\Omega)$ and there exists a Borel set $N \subset \Omega$ with $\mathcal{H}^{N-1}(N)=0$ such that $\varphi(\cdot, \xi)$ is approximately continuous in $\Omega \backslash N$ for all $\xi \in R^{N}$; 
$\left(i_{4}\right)$ there exists a positive constant $M$ such that for all $\xi \in \mathbb{R}^{N}$

$$
\int_{\Omega}\left|\nabla_{x} \varphi(x, \xi)\right| d x \leq M
$$

$\left(i_{5}\right)$ for every $t \in K$ the map $r \mapsto \vartheta(r, t)$ belongs to $C^{1}(\Omega)$, there exists a positive constant $C$ such that $\left|\nabla_{r} \vartheta(r, t)\right| \leq C$ for every $t, r \in K$ and there exists a modulus of continuity $\widetilde{\omega}$ such that

$$
\left|\nabla_{r} \vartheta(r, t)-\nabla_{r} \vartheta\left(r^{\prime}, t\right)\right| \leq \widetilde{\omega}\left(\left|r-r^{\prime}\right|\right)
$$

for all $t, r, r^{\prime} \in K$.

Then $\phi$ is a NA jointly convex function.

Proof. By Proposition 2.2 there exists a sequence $\left\{\alpha_{k}\right\} \subset C_{c}^{\infty}\left(\mathbb{R}^{N}\right)$, with $\alpha_{k} \geq 0$ and $\int_{\mathbb{R}^{N}} \alpha_{k}(\xi) d \xi=1$ such that,

$$
\varphi(x, \xi)=\sup _{k \in \mathbb{N}}\left\langle a_{k}(x), \xi\right\rangle
$$

where for every $i=1, \cdots, N$

$$
a_{i, k}(x)=-\int_{\mathbb{R}^{N}} \varphi(x, \xi) \frac{\partial}{\partial \xi_{i}} \alpha_{k}(\xi) d \xi=\int_{\mathbb{R}^{N}} \frac{\partial}{\partial \xi_{i}} \varphi(x, \xi) \alpha_{k}(\xi) d \xi
$$

and $a_{k}=\left(a_{1, k}, \ldots, a_{N, k}\right)$. By $\left(i_{2}\right)$ the functions $a_{k}$ are bounded and by $\left(i_{3}\right)$ and $\left(i_{4}\right)$ the functions $a_{k}$ belong to $W^{1,1}\left(\Omega ; \mathbb{R}^{N}\right)$ and so there exists a Borel set $N \subset \Omega$ with $\mathcal{H}^{N-1}(N)=0$ such that $a_{k}$ are approximately continuous in $\Omega \backslash N$. As in Example 5.23 (a) of [13], we can choose a countable dense sequence $c_{h}$ in $K$ such that

$$
\phi(x, r, t, \xi)=\vartheta(r, t) \varphi(x, \xi)=\sup _{h, k \in \mathbb{N}}\left[\vartheta\left(r, c_{h}\right)-\vartheta\left(t, c_{h}\right)\right]\left\langle a_{k}(x), \xi\right\rangle .
$$

Then the functions

$$
g_{h, k}(x, r):=\vartheta\left(r, c_{h}\right) a_{k}(x)
$$

satisfy the conditions (a), (b), (c), (d) and (e) (or (A), (B) and (C) in the scalar case). 
A first lower semicontinuity result can be obtained for NA jointly convex integrands in the vectorial case.

Theorem 3.4. Let $K \subset \mathbb{R}^{m}$ be a compact set and let $\phi: \Omega \times K \times K \times \mathbb{R}^{N} \rightarrow$ $[0,+\infty)$ be a locally bounded NA jointly convex function. Then, for every $\left\{u_{n}\right\} \subset \operatorname{SBV}^{p}\left(\Omega ; \mathbb{R}^{m}\right)$ and $u \in \operatorname{SBV}^{p}\left(\Omega ; \mathbb{R}^{m}\right)$ such that $u_{n}(x) \rightarrow u(x)$ for $\mathcal{L}^{N}$-a.e. $x \in \Omega, u_{n}(x), u(x) \in K$ for $\mathcal{L}^{N}$-a.e. $x \in \Omega$ and

$$
\sup _{n \in \mathbb{N}}\left[\left\|u_{n}\right\|_{\infty}+\int_{\Omega}\left|\nabla u_{n}\right|^{p} d x+\mathcal{H}^{N-1}\left(J_{u_{n}}\right)\right]<+\infty,
$$

we have

$$
\int_{\Omega \cap J_{u}} \phi\left(x, u^{-}, u^{+}, \nu_{u}\right) d \mathcal{H}^{N-1} \leq \liminf _{n \rightarrow+\infty} \int_{\Omega \cap J_{u_{n}}} \phi\left(x, u_{n}^{-}, u_{n}^{+}, \nu_{u_{n}}\right) d \mathcal{H}^{N-1} .
$$

Proof. We follow the outlines of the proof of Theorem 5.22 in [13].

Let

$$
C:=\sup _{n \in \mathbb{N}}\left[\left\|u_{n}\right\|_{\infty}+\int_{\Omega}\left|\nabla u_{n}\right|^{p} d x+\mathcal{H}^{N-1}\left(J_{u_{n}}\right)\right] .
$$

Since $\phi$ is nonnegative, we have

$$
\phi(x, r, t, \xi)=\sup _{j \in \mathbb{N}}\left\langle g_{j}(x, r)-g_{j}(x, t), \xi\right\rangle^{+}
$$

for all $(x, r, t, \xi) \in \Omega \times K \times K \times \mathbb{R}^{N}$. By Lemma 2.1, it is enough to prove the lower semicontinuity for functionals of the type

$$
F_{g}(u):=\int_{J_{u}}\left\langle g\left(x, u^{+}\right)-g\left(x, u^{-}\right), \nu_{u}\right\rangle^{+} d \mathcal{H}^{N-1} .
$$

Let us now fix $\psi \in C_{0}^{1}(\Omega), 0 \leq \psi \leq 1$. The lower semicontinuity of the functional in (3.4) will follow if we prove the continuity of

$$
F_{g}^{\psi}(u):=\int_{J_{u}}\left\langle g\left(x, u^{+}\right)-g\left(x, u^{-}\right), \nu_{u}\right\rangle \psi(x) d \mathcal{H}^{N-1} .
$$

Using the chain rule formula (2.6) we have

$$
\int_{J_{u}}\left\langle g\left(x, u^{+}\right)-g\left(x, u^{-}\right), \nu_{u}\right\rangle \psi d \mathcal{H}^{N-1}=-\int_{\Omega}\langle\nabla \psi(x), g(x, u(x))\rangle d x
$$




$$
-\int_{\Omega} \psi(x) \operatorname{div}_{x} g(x, u(x)) d x-\int_{\Omega} \psi(x) \operatorname{tr}\left[\nabla_{r} g(x, u(x)) \cdot \nabla u(x)\right] d x .
$$

Notice that

$$
\begin{aligned}
& \int_{\Omega}\langle\nabla \psi(x), g(x, u(x))\rangle d x=\lim _{n \rightarrow+\infty} \int_{\Omega}\left\langle\nabla \psi(x), g\left(x, u_{n}(x)\right)\right\rangle d x ; \\
& \int_{\Omega} \psi(x) \operatorname{div}_{x} g(x, u(x)) d x=\lim _{n \rightarrow+\infty} \int_{\Omega} \psi(x) \operatorname{div}_{x} g\left(x, u_{n}(x)\right) d x ; \\
& \int_{\Omega} \psi(x) \operatorname{tr}\left[\nabla_{r} g(x, u(x)) \cdot \nabla u(x)\right] d x \\
= & \lim _{n \rightarrow+\infty} \int_{\Omega} \psi(x) \operatorname{tr}\left[\nabla_{r} g\left(x, u_{n}(x)\right) \cdot \nabla u_{n}(x)\right] d x .
\end{aligned}
$$

In fact, by using (d), the sequence $\left\{g\left(x, u_{n}\right)-g(x, u)\right\}$ converges almost everywhere to 0 and is equibounded in $L^{\infty}(\Omega)$. Similarly, by using (b), $\left\{\operatorname{div}_{x} g\left(x, u_{n}\right)\right\}$ converges almost everywhere to $\operatorname{div}_{x} g(x, u)$ and is equibounded by an $L^{1}$-function. Thus (3.6) and (3.7) hold. In order to prove equality (3.8), we observe that, by using (e), $\psi \in L^{\infty}(\Omega)$, $\nabla_{r} g\left(x, u_{n}\right) \rightarrow \nabla_{r} g(x, u)$ strongly in $L^{p^{\prime}}\left(\Omega ; M I^{N \times m}\right)$ and $\nabla u_{n} \rightarrow \nabla u$ weakly in $L^{p}\left(\Omega ; M^{m \times N}\right)$. By (3.6), (3.7) and (3.8) we have the continuity of the functional $F_{g}^{\psi}$ and so the lower semicontinuity of $F_{g}$.

The same lower semicontinuity result holds for NA jointly convex integrands in the scalar case $(m=1)$, by repeating the proof and by using the scalar chain rule (2.9) (Theorem 2.7) instead of the vectorial chain rule (2.6) (Theorem 2.6).

\section{Nonautonomous $B V$ or $W^{1,1}$ Jointly COnVEX Functions}

In this section we give a different definition of nonautonomous jointly convex function with $B V$ (or $W^{1,1}$ ) dependence with respect to the spatial variable $x$.

Let $K \subset \mathbb{R}^{m}$ be a compact set and let $\phi: \Omega \times K \times K \times \mathbb{R}^{N} \rightarrow[0,+\infty)$ be a locally bounded function.

Definition 4.1. The function $\phi$ is $B V$ jointly convex (respectively $W^{1,1}$ jointly convex) if the following conditions hold 
$\left(\alpha^{\prime}\right)$ for every $(r, t, \xi) \in K \times K \times \mathbb{R}^{N}$ the function $\phi(\cdot, r, t, \xi)$ belongs to $B V$ and there exists a Borel set $N \subset \Omega$ with $\mathcal{H}^{N-1}(N)=0$ such that $\phi(\cdot, r, t, \xi)=\phi^{-}(\cdot, r, t, \xi)$ (respectively $\phi(\cdot, r, t, \xi)=\widetilde{\phi}(\cdot, r, t, \xi)$ ) in $\Omega \backslash N$ for all $(r, t, \xi) \in K \times K \times \mathbb{R}^{N}$;

$\left(\beta^{\prime}\right)$ for every $x \in \Omega \backslash N$ the function $\phi(x, \cdot, \cdot, \cdot)$ is jointly convex;

$\left(\gamma^{\prime}\right)$ there exists a positive constant $L$ such that

$$
\left|\phi(x, r, t, \xi)-\phi\left(x, r^{\prime}, t, \xi\right)\right| \leq L\left|r-r^{\prime}\right|
$$

for all $x \in \Omega \backslash N$, for all $r, r^{\prime}, t \in K$ and $\xi \in \mathbb{R}^{N}$.

Remark 4.2. We will prove (see Theorem 4.8 below) that for integrand $B V$ jointly convex the lower semicontinuity holds by requiring the further condition that $\phi$ is strictly positive for $\mathcal{H}^{N-1}$ almost everywhere $x \in \Omega$.

Remark 4.3. We give some examples of $B V$ jointly convex functions.

The model case is

$$
\phi(x, r, t, \xi):=\langle g(x, r)-g(x, t), \xi\rangle^{+},
$$

with $g$ satisfying the following conditions

$\left(\alpha^{\prime \prime}\right)$ for every $r \in K$ the function $g(\cdot, r)$ is a locally bounded $B V$ and there exists a Borel set $N \subset \Omega$ with $\mathcal{H}^{N-1}(N)=0$ such that $g(\cdot, r)=g^{-}(\cdot, r)$ in $\Omega \backslash N$ for all $r \in K$;

$\left(\gamma^{\prime \prime}\right)$ there exists a positive constant $L$ such that

$$
\left|g(x, r)-g\left(x, r^{\prime}\right)\right| \leq L\left|r-r^{\prime}\right|
$$

for all $x \in \Omega \backslash N$, for all $r, r^{\prime} \in K$.

Another example is

$$
\phi(x, r, t, \xi):=a(x) \varphi(r, t, \xi),
$$

where $a$ is a nonnegative bounded $B V$ function coinciding with its lower approximate limit $a^{-}$and $\varphi$ is a jointly convex function satisfying the following condition:

there exists a positive constant $L$ such that

$$
\left|\varphi(r, t, \xi)-\varphi\left(r^{\prime}, t, \xi\right)\right| \leq L\left|r-r^{\prime}\right|
$$

for all $r, r^{\prime}, t \in K$ and $\xi \in \mathbb{R}^{N}$.

Moreover let

$$
\phi(x, r, t, \xi):=\gamma(|r-t|) \varphi(x, \xi),
$$


where $\gamma:[0,+\infty[\rightarrow[0,+\infty[$ is a continuous, increasing and subadditive function with $\gamma(0)=0$ and $\gamma(s) \leq C|s|$ for all $s \in \mathbb{R}$, and $\varphi$ is a bounded function, which is convex, positively 1-homogeneous and even in $\xi$ and it satisfies conditions $\left(i_{3}\right)$ below.

Functions of the type (A2) are already considered in [5].

Another type of $B V$ jointly convex function, which generalizes example (A2), can be obtained as in the following way.

Let $\phi: \Omega \times K \times K \times \mathbb{R}^{N} \rightarrow[0,+\infty)$

$$
\phi(x, r, t, \xi):=\vartheta(r, t) \varphi(x, \xi)
$$

where

$\left(i_{1}\right) \vartheta$ is a Lipschitz continuous function and it is a pseudo-distance in $K$ (i.e. a positive, symmetric function satisfying the triangle inequality);

$\left(i_{2}\right) \varphi$ is a bounded function and it is convex, positively 1-homogeneous and even in $\xi$

$\left(i_{3}\right)$ for every $\xi \in \mathbb{R}^{N}$ the map $x \mapsto \varphi(x, \xi)$ belongs to $B V$ and there exists a Borel set $N \subset \Omega$ with $\mathcal{H}^{N-1}(N)=0$ such that $\varphi(\cdot, \xi)$ coincides with its lower approximate limit $\varphi^{-}(\cdot, \xi)$ in $\Omega \backslash N$ for all $\xi \in R^{N}$.

In order to study the lower semicontinuity, firstly we consider the model case

$$
\phi(x, r, t, \xi):=a(x) \varphi(r, t, \xi),
$$

where $\varphi$ is a jointly convex function and $a$ is a locally bounded $B V$ function.

Proposition 4.4. Let $a: \Omega \rightarrow[0,+\infty)$ be a locally bounded $B V$ function coinciding with its lower approximate limit $a^{-}$and let $\varphi: K \times K \times \mathbb{R}^{N} \rightarrow$ $[0,+\infty)$ be a locally bounded jointly convex function. Then, for every $\left\{u_{n}\right\} \subset \operatorname{SBV}^{p}\left(\Omega ; \mathbb{R}^{m}\right)$ and $u \in \operatorname{SBV}^{p}\left(\Omega ; \mathbb{R}^{m}\right)$ such that $u_{n}(x) \rightarrow u(x)$ for $\mathcal{L}^{N}$-a.e. $x \in \Omega, u_{n}(x), u(x) \in K$ for $\mathcal{L}^{N}$-a.e. $x \in \Omega$ and

$$
\sup _{n \in \mathbb{N}}\left[\left\|u_{n}\right\|_{\infty}+\int_{\Omega}\left|\nabla u_{n}\right|^{p} d x+\mathcal{H}^{N-1}\left(J_{u_{n}}\right)\right]<+\infty,
$$

we have

$$
\int_{\Omega \cap J_{u}} a(x) \varphi\left(u^{-}, u^{+}, \nu_{u}\right) d \mathcal{H}^{N-1} \leq \liminf _{n \rightarrow+\infty} \int_{\Omega \cap J_{u_{n}}} a(x) \varphi\left(u_{n}^{-}, u_{n}^{+}, \nu_{u_{n}}\right) d \mathcal{H}^{N-1} .
$$


Proof. It suffices to note that by Theorem 2.4 the function $a$ is lower semicontinuous with respect to the 1-capacity. Therefore the conclusion of the proof is obtained by using Lemma 2.5, Proposition 3.1 in [5] and Lemma 2.1 .

In order to treat the general case of $B V$ jointly convex function firstly we study integrands which are lower semicontinuous in $x$ uniformly with respect to the other variables. For these integrands the following approximation from below holds with functions of the type (4.1).

Proposition 4.5. Let $\phi: \Omega \times K \times K \times \mathbb{R}^{N} \rightarrow[0,+\infty)$ be a locally bounded Borel function such that

$(\mathcal{A})$ given $x_{0} \in \Omega$, for all $\varepsilon>0$ there exists $\delta>0$ such that

$$
\phi\left(x_{0}, r, t, \xi\right) \leq(1+\varepsilon) \phi(x, r, t, \xi)
$$

for all $(x, r, t, \xi) \in \Omega \times K \times K \times \mathbb{R}^{N}$ such that $\left|x-x_{0}\right|<\delta$;

$(\beta)$ for every $x \in \Omega$ the function $\phi(x, \cdot, \cdot, \cdot)$ is jointly convex.

Then there exists $a_{j} \in C_{0}^{\infty}(\Omega), 0 \leq a_{j} \leq 1, a_{j}\left(x_{j}\right)=1$ for some $x_{j} \in \Omega$, and there exists $g_{j} \in \mathcal{C}\left(K ; \mathbb{R}^{N}\right)$ such that

$$
\phi(x, r, t, \xi)=\sup _{j \in \mathbb{N}} a_{j}(x)\left\langle g_{j}(r)-g_{j}(t), \xi\right\rangle^{+}
$$

for all $(x, r, t, \xi) \in \Omega \times K \times K \times \mathbb{R}^{N}$.

Proof. We adapt the proof of Proposition 6.40 of [27] (proven in [19]). Let $\mathcal{G}$ be the class of all functions $G: \Omega \times K \times K \times \mathbb{R}^{N} \rightarrow[0,+\infty)$ of the form

$$
G(x, r, t, \xi)=\varphi(x)\langle g(r)-g(t), \xi\rangle^{+} \quad \forall(x, r, t, \xi) \in \Omega \times K \times K \times \mathbb{R}^{N},
$$

with $g \in \mathcal{C}\left(K ; \mathbb{R}^{N}\right), \varphi \in C_{0}^{\infty}(\Omega), 0 \leq \varphi \leq 1, \varphi(x)=1$ for some $x \in \Omega$, and

$$
G(x, r, t, \xi) \leq \phi(x, r, t, \xi) \quad \forall(x, r, t, \xi) \in \Omega \times K \times K \times \mathbb{R}^{N} .
$$

We remark that $\mathcal{G} \neq \emptyset$, since, for $g=0$, we have $G=0 \in \mathcal{G}$.

We will prove that

(4.4) $\phi(x, r, t, \xi)=\sup _{G \in \mathcal{G}} G(x, r, t, \xi) \quad$ for all $(x, r, t, \xi) \in \Omega \times K \times K \times \mathbb{R}^{N}$.

The inequality

$$
\sup _{G \in \mathcal{G}} G(x, r, t, \xi) \leq \phi(x, r, t, \xi)
$$


is due to the definition of $\mathcal{G}$. Now, given $x_{0} \in \Omega$, we will prove the opposite inequality

$$
\phi\left(x_{0}, r, t, \xi\right) \leq \sup _{G \in \mathcal{G}} G\left(x_{0}, r, t, \xi\right) .
$$

By using $(\mathcal{A})$, for all $\varepsilon>0$ there exists $\delta>0$ such that (4.3) holds. Let $\varphi \in C_{0}^{\infty}(\Omega), 0 \leq \varphi \leq 1, \varphi=1$ on $B\left(x_{0}, \delta / 2\right)$ and $\varphi=0$ outside $B\left(x_{0}, \delta\right)$. Since the function $\phi\left(x_{0}, \cdot, \cdot, \cdot\right)$ is jointly convex, there exists a sequence of functions $g_{k} \in \mathcal{C}\left(K ; \mathbb{R}^{N}\right)$ such that

$$
\phi\left(x_{0}, r, t, \xi\right)=\sup _{k \in \mathbb{N}}\left\langle g_{k}(r)-g_{k}(t), \xi\right\rangle^{+} \quad \text { for all }(r, t, \xi) \in K \times K \times \mathbb{R}^{N} .
$$

For every $\epsilon>0$, if we define

$$
G_{k}^{\epsilon}(x, r, t, \xi):=\varphi(x)\left\langle(1-\epsilon)\left(g_{k}(r)-g_{k}(t)\right), \xi\right\rangle^{+}
$$

for every $(x, r, t, \xi) \in \Omega \times K \times K \times \mathbb{R}^{N}$, then $G_{k}^{\epsilon} \leq \phi, G_{k}^{\epsilon} \in \mathcal{G}$ and

$$
(1-\epsilon) \phi\left(x_{0}, r, t, \xi\right)=\sup _{k \in \mathbb{N}} G_{k}^{\epsilon}\left(x_{0}, r, t, \xi\right) \leq \sup _{G \in \mathcal{G}} G\left(x_{0}, r, t, \xi\right)
$$

hence, by letting $\epsilon \rightarrow 0^{+}$, (4.4) is obtained. By Lemma 3.2 of [4] there exists a sequence $G_{j}$ in $\mathcal{G}$ such that

$$
\begin{gathered}
G_{j}(x, r, t, \xi)=a_{j}(x)\left\langle g_{j}(r)-g_{j}(t), \xi\right\rangle^{+} \\
\phi(x, r, t, \xi)=\sup _{j \in \mathbb{N}} G_{j}(x, r, t, \xi),
\end{gathered}
$$

for every $(x, r, t, \xi) \in \Omega \times K \times K \times \mathbb{R}^{N}$.

Proposition 4.6. Let $\phi: \Omega \times K \times K \times \mathbb{R}^{N} \rightarrow[0,+\infty)$ be a locally bounded Borel function such that condition $(\mathcal{A})$ and $(\beta)$ hold. Then, for every $\left\{u_{n}\right\} \subset \operatorname{SBV}^{p}\left(\Omega ; \mathbb{R}^{m}\right)$ and $u \in \operatorname{SBV}^{p}\left(\Omega ; \mathbb{R}^{m}\right)$ such that $u_{n}(x) \rightarrow u(x)$ for $\mathcal{L}^{N}$-a.e. $x \in \Omega, u_{n}(x), u(x) \in K$ for $\mathcal{L}^{N}$-a.e. $x \in \Omega$ and

$$
\sup _{n \in \mathbb{N}}\left[\left\|u_{n}\right\|_{\infty}+\int_{\Omega}\left|\nabla u_{n}\right|^{p} d x+\mathcal{H}^{N-1}\left(J_{u_{n}}\right)\right]<+\infty
$$

we have

$$
\int_{\Omega \cap J_{u}} \phi\left(x, u^{-}, u^{+}, \nu_{u}\right) d \mathcal{H}^{N-1} \leq \liminf _{n \rightarrow+\infty} \int_{\Omega \cap J_{u_{n}}} \phi\left(x, u_{n}^{-}, u_{n}^{+}, \nu_{u_{n}}\right) d \mathcal{H}^{N-1} .
$$


Proof. By Proposition 4.5, we have that there exist $\left\{a_{j}\right\} \subset C_{0}^{\infty}(\Omega), 0 \leq$ $a_{j} \leq 1$, and $g_{j} \in \mathcal{C}\left(K ; \mathbb{R}^{N}\right)$ such that

$$
\phi(x, r, t, \xi)=\sup _{j \in \mathbb{N}} a_{j}(x)\left\langle g_{j}(r)-g_{j}(t), \xi\right\rangle^{+}
$$

for all $(x, r, t, \xi) \in \Omega \times K \times K \times \mathbb{R}^{N}$. For $j \in \mathbb{N}$, the function

$$
\phi_{j}: \Omega \times K \times K \times \mathbb{R}^{N} \rightarrow[0,+\infty)
$$

defined by $\phi_{j}(x, r, t, \xi):=a_{j}(x)\left\langle g_{j}(r)-g_{j}(t), \xi\right\rangle^{+}$satisfies the assumptions of Proposition 3.1 in [5]). Therefore, the corresponding functionals are all lower semicontinuous and the thesis follows by Lemma 2.1.

As in Theorem 3.4 of [2], it is possible to obtain the lower semicontinuity by assuming, instead of hypothesis $(\mathcal{A})$, some conditions which are easier to verify.

Proposition 4.7. Let $\phi: \Omega \times K \times K \times \mathbb{R}^{N} \rightarrow[0,+\infty)$ be a locally bounded Borel function such that

$\left(\mathcal{A}_{1}\right) \phi(\cdot, \cdot, \cdot, \xi)$ is lower semicontinuous on $\Omega \times K \times K$ for every $\xi \in \mathbb{R}^{N}$;

$\left(\mathcal{A}_{2}\right) \phi(x, r, t, \xi)>0 \quad \forall(x, r, t, \xi) \in\left(\Omega \backslash N_{0}\right) \times K \times K \times\left(\mathbb{R}^{N} \backslash\{0\}\right)$ with $\mathcal{H}^{N-1}\left(N_{0}\right)=0$;

$(\beta)$ for every $x \in \Omega$ the function $\phi(x, \cdot, \cdot, \cdot)$ is jointly convex;

$\left(\gamma^{\prime}\right)$ there exists a positive constant $L$ such that

$$
\left|\phi(x, r, t, \xi)-\phi\left(x, r^{\prime}, t, \xi\right)\right| \leq L\left|r-r^{\prime}\right|
$$

for all $x \in \Omega$, for all $r, r^{\prime}, t \in K$ and $\xi \in \mathbb{R}^{N}$.

Then condition $(\mathcal{A})$ holds.

Proof. Notice that, since $\phi$ is locally bounded and positively 1-homogeneous with respect to $\xi$, for any open set $\Omega^{\prime} \subset \subset \Omega$, there exists a constant $\Lambda^{\prime}$ such that

$$
0 \leq \phi(x, r, t, \xi) \leq \Lambda^{\prime}|\xi| \quad \text { for all }(x, r, t, \xi) \in \Omega^{\prime} \times K \times K \times \mathbb{R}^{N} .
$$

Hence the convexity of $\phi$ with respect to $\xi$ immediately yields that, for all $\left(x, r, t, \xi_{1}\right),\left(x, r, t, \xi_{2}\right) \in \Omega^{\prime} \times K \times K \times \mathbb{R}^{N}$,

$$
\left|\phi\left(x, r, t, \xi_{1}\right)-\phi\left(x, r, t, \xi_{2}\right)\right| \leq \Lambda^{\prime}\left|\xi_{1}-\xi_{2}\right| .
$$

Then $\phi$ is lower semicontinuous in $\Omega^{\prime} \times K \times K \times \mathbb{R}^{N}$ and $\phi(x, \cdot, \cdot, \cdot)$ is continuous in $K \times K \times \mathbb{R}^{N}$ for every $x \in \Omega^{\prime}$. 
We claim that, given $x_{0} \in \Omega^{\prime} \backslash N_{0}$, for all $\varepsilon>0$, condition $(\mathcal{A})$ holds, i.e. there exists $\delta>0$ such that

$$
\phi\left(x_{0}, r, t, \xi\right) \leq(1+\varepsilon) \phi(x, r, t, \xi)
$$

for all $(x, r, t, \xi) \in \Omega^{\prime} \times K \times K \times \mathbb{R}^{N}$ such that $\left|x-x_{0}\right|<\delta$.

To prove this, we argue by contradiction, assuming that there exist $x_{0} \in$ $\Omega^{\prime} \backslash N_{0}$ and $\varepsilon_{0}>0$ such that for any $k \in \mathbb{N}$, there exist sequences $\left\{x_{k}\right\} \subseteq \Omega^{\prime}$, with $\left|x_{k}-x_{0}\right|<1 / k$, and $\left\{\left(r_{k}, t_{k}, \xi_{k}\right)\right\} \subseteq K \times K \times \mathbb{R}^{N}$ such that

$$
\phi\left(x_{0}, r_{k}, t_{k}, \xi_{k}\right)>\left(1+\varepsilon_{0}\right) \phi\left(x_{k}, r_{k}, t_{k}, \xi_{k}\right) .
$$

Clearly, by the positive 1-homogeneity of $\phi(x, r, t, \cdot)$, we may assume that $\left|\xi_{k}\right|=1$, for every $k \in \mathbb{N}$; hence, up to a subsequence, there exists $\xi_{0} \in$ $\mathbb{S}^{N-1}$ such that $\xi_{k} \rightarrow \xi_{0}$. Moreover, since $\left\{s_{k}\right\},\left\{t_{k}\right\} \subseteq K$, we may assume that also $s_{k} \rightarrow s_{0}, t_{k} \rightarrow t_{0}$, with $s_{0}, t_{0} \in K$. Then, passing to the limit when $k \rightarrow+\infty$ in (4.11) and using the lower semicontinuity of $\phi$ and the continuity of $\phi\left(x_{0}, \cdot, \cdot, \cdot\right)$, we get that

$$
\begin{gathered}
\phi\left(x_{0}, r_{0}, t_{0}, \xi_{0}\right)=\lim _{k \rightarrow+\infty} \phi\left(x_{0}, r_{k}, t_{k}, \xi_{k}\right) \\
\geq\left(1+\varepsilon_{0}\right) \liminf _{k \rightarrow+\infty} \phi\left(x_{k}, r_{k}, t_{k}, \xi_{k}\right) \geq\left(1+\varepsilon_{0}\right) \phi\left(x_{0}, r_{0}, t_{0}, \xi_{0}\right) .
\end{gathered}
$$

Hence, $\phi\left(x_{0}, r_{0}, t_{0}, \xi_{0}\right)=0$, which is a contradiction, since $x_{0} \in \Omega^{\prime} \backslash N_{0}$; therefore (4.10) holds.

The conclusion follows by letting $\Omega^{\prime} \nearrow \Omega$.

Theorem 4.8. Let $\phi$ be a $B V$ jointly convex function satisfying $\left(\mathcal{A}_{2}\right)$. Then the lower semicontinuity (4.6) holds.

Proof. Firstly, we claim that for every open set $\Omega^{\prime} \subset \subset \Omega$, for every $h \in \mathbb{N}$ there exists an open set $A_{h} \subset \Omega^{\prime}$ with $N_{0} \cap \Omega^{\prime} \subset A_{h}, C_{1}\left(A_{h}\right)<1 / h$, such that the function $\phi$ is lower semicontinuous in $\left(\Omega^{\prime} \backslash A_{h}\right) \times K \times K \times \mathbb{R}^{N}$ and, given $x_{0} \in \Omega^{\prime} \backslash N_{0}$, for all $\varepsilon>0$ there exists $\delta>0$ such that

$$
\phi\left(x_{0}, r, t, \xi\right) \leq(1+\varepsilon) \phi(x, r, t, \xi)
$$

for all $(x, r, t, \xi) \in\left(\Omega^{\prime} \backslash A_{h}\right) \times K \times K \times \mathbb{R}^{N}$ such that $\left|x-x_{0}\right|<\delta$. As in the proof of Theorem 4.7, conditions (4.8) and (4.9) hold in $\left(\Omega^{\prime} \backslash N_{0}\right) \times$ $K \times K \times \mathbb{R}^{N}$. Let us now fix $h$, a sequence $\left\{\xi_{j}\right\}$ dense in $\mathbb{R}^{N}$ and two sequences $\left\{r_{j}\right\}$ and $\left\{t_{j}\right\}$ dense in $K$. By Theorem 2.4 for every $j \in \mathbb{N}$ the function $\phi\left(\cdot, r_{j}, t_{j}, \xi_{j}\right)$ is $C^{1}$-quasi lower semicontinuous; then for all $j$ there exists an open set $A_{j, h} \subset \Omega^{\prime}, N_{0} \subset A_{j, h}$, with $C_{1}\left(A_{j, h}\right)<1 /\left(h 2^{j}\right)$, such that 
$\phi\left(\cdot, r_{j}, t_{j}, \xi_{j}\right)$ is lower semicontinuous in $\Omega^{\prime} \backslash A_{j, h}$. Setting $A_{h}=\cup_{j} A_{j, h}, A_{h}$ is open, $C_{1}\left(A_{h}\right)<1 / h$, and making use of $(4.9)$ and $\left(\gamma^{\prime}\right)$, one easily gets that $\phi(\cdot, r, t, \xi)$ is lower semicontinuous in $\Omega^{\prime} \backslash A_{h}$ for every $(r, t, \xi) \in K \times K \times \mathbb{R}^{N}$ and $\phi(\cdot, \cdot, \cdot, \xi)$ is lower semicontinuous in $\left(\Omega^{\prime} \backslash A_{h}\right) \times K \times K$ for every $\xi \in \mathbb{R}^{N}$ (we can assume that $A_{h}$ is a decreasing sequence of open sets). Hence, as in the proof of Theorem 4.7, the claim holds.

Therefore by Proposition 4.5 there exist $\left\{a_{j}^{h}\right\} \subset C_{0}^{\infty}\left(\Omega^{\prime}\right), 0 \leq a_{j}^{h} \leq 1$, and $g_{j}^{h} \in \mathcal{C}\left(K ; \mathbb{R}^{N}\right)$ such that

$$
\phi(x, r, t, \xi)=\sup _{j \in \mathbb{N}} a_{j}(x)\left\langle g_{j}^{h}(r)-g_{j}^{h}(t), \xi\right\rangle^{+}
$$

for all $(x, r, t, \xi) \in\left(\Omega^{\prime} \backslash A_{h}\right) \times K \times K \times \mathbb{R}^{N}$. Moreover for every $j \in \mathbb{N}$ there exists $x_{j}^{h} \in \Omega^{\prime} \backslash A_{h}$ such that $a_{j}^{h}\left(x_{j}^{h}\right)=1$. If we set

$$
\psi_{j}^{h}(r, t, \xi)=\left\langle g_{j}^{h}(r)-g_{j}^{h}(t), \xi\right\rangle^{+},
$$

we have that $\psi_{j}^{h} \geq 0, \psi_{j}^{h}$ is a locally bounded jointly convex function and

$$
\phi(x, r, t, \xi)=\sup _{j \in \mathbb{N}} a_{j}^{h}(x) \psi_{j}^{h}(r, t, \xi)
$$

for all $(x, r, t, \xi) \in\left(\Omega^{\prime} \backslash A_{h}\right) \times K \times K \times \mathbb{R}^{N}$.

We will prove that there exists a constant $C>0$ (independent of $h$ ) such that

$$
\sup _{j \in \mathbb{N}} \psi_{j}^{h}(r, t, \nu) \leq C \quad \forall(r, t, \nu) \in K \times K \times \mathbb{S}^{N-1} .
$$

Since $\phi$ is locally bounded, there exists a constant $C>0$ such that $\phi(x, r, t, \nu) \leq C$ for every $(x, r, t, \nu) \in \Omega^{\prime} \times K \times K \times \mathbb{S}^{N-1}$. Then for every $(r, t, \nu) \in K \times K \times \mathbb{S}^{N-1}$ and for every $j, h \in \mathbb{N}$ we have

$$
\psi_{j}^{h}(r, t, \nu)=a_{j}^{h}\left(x_{j}^{h}\right) \psi_{j}^{h}(r, t, \nu) \leq \phi\left(x_{j}^{h}, r, t, \nu\right) \leq C .
$$

Then (4.13) holds.

Let $\varphi_{h} \in W^{1,1}\left(\mathbb{R}^{N}\right)$ be a capacitary quasi-potential of $A_{h}$. More precisely, let us assume that there exists a Borel set $N_{h} \subset \mathbb{R}^{N}$, with $C_{1}\left(N_{h}\right)=$ $\mathcal{H}^{N-1}\left(N_{h}\right)=0$, such that $0 \leq \widetilde{\varphi}_{h}(x) \leq 1$ for every $x \in \mathbb{R}^{N} \backslash N_{h}, \widetilde{\varphi}_{h}=1$ on $A_{h} \backslash N_{h}$,

$$
\int_{\mathbb{R}^{N}}\left|\nabla \widetilde{\varphi}_{h}\right| d x \leq C_{1}\left(A_{h}\right)+\frac{1}{h}<\frac{2}{h}
$$


and by Lemma 1.2 in [16], $\widetilde{\varphi}_{h}(x)$ tends to 0 , as $h \rightarrow+\infty$, for $\mathcal{H}^{N-1}$ almost every $x \in \Omega$. Moreover, setting $\widetilde{N}=\cup_{h} N_{h}, C_{1}(\widetilde{N})=\mathcal{H}^{N-1}(\widetilde{N})=0$, for every $j \in \mathbb{N}$ and for every $x \in \Omega^{\prime} \backslash \widetilde{N}$ we set

$$
\widetilde{\alpha}_{j}^{h}(x):=\max \left\{0, a_{j}^{h}(x)-\widetilde{\varphi}_{h}(x)\right\} .
$$

Since $0 \leq \widetilde{\varphi}_{h}(x) \leq 1$, we have

$$
0 \leq \widetilde{\alpha}_{j}^{h}(x) \leq 1, \quad a_{j}^{h}(x) \geq \widetilde{\alpha}_{j}^{h}(x) \geq a_{j}^{h}(x)-\widetilde{\varphi}_{h}(x) \quad \text { for all } x \in \Omega^{\prime}
$$

and

$$
\phi(x, r, t, \xi) \geq \widetilde{\alpha}_{j}^{h}(x) \psi_{j}^{h}(r, t, \xi)
$$

for all $(x, r, t, \xi) \in \Omega^{\prime} \times K \times K \times \mathbb{R}^{N}$. Finally, we set for all $h, j \in \mathbb{N}$

$$
\phi_{j}^{h}(x, r, t, \xi)=\widetilde{\alpha}_{j}^{h}(x) \psi_{j}^{h}(r, t, \xi), \quad \phi^{h}(x, r, t, \xi)=\sup _{j \in \mathbb{N}} \phi_{j}^{h}(x, r, t, \xi),
$$

for all $(x, r, t, \xi) \in \Omega^{\prime} \times K \times K \times \mathbb{R}^{N}$. We notice that $\phi_{j}^{h}$ satisfies all the assumptions of Proposition 4.4. Hence the corresponding functional

$$
\mathcal{F}_{\phi_{j}^{h}}(u):=\int_{\Omega^{\prime} \cap J_{u}} \phi_{j}^{h}\left(x, u^{-}, u^{+}, \nu_{u}\right) d \mathcal{H}^{N-1}
$$

is lower semicontinuous; by Lemma 2.1 the same holds for the functional

$$
\mathcal{F}_{\phi^{h}}(u):=\int_{\Omega^{\prime} \cap J_{u}} \phi^{h}\left(x, u^{-}, u^{+}, \nu_{u}\right) d \mathcal{H}^{N-1} .
$$

Moreover, since

$$
\phi_{j}^{h}(x, r, t, \xi) \geq\left[a_{j}^{h}(x)-\widetilde{\varphi}_{h}(x)\right] \psi_{j}^{h}(r, t, \xi)
$$

for all $(x, r, t, \xi) \in \Omega^{\prime} \times K \times K \times \mathbb{R}^{N}$, we have that

$$
\phi^{h}(x, r, t, \xi) \geq \phi(x, r, t, \xi)-\widetilde{\varphi}_{h}(x) \psi^{h}(r, t, \xi)
$$

for all $(x, r, t, \xi) \in \Omega^{\prime} \times K \times K \times \mathbb{R}^{N}$, where $\psi^{h}:=\sup _{j \in \mathbb{N}} \psi_{j}^{h}$; by (4.13) there exists a constant $C>0$ such that

$$
0 \leq \psi^{h}(r, t, \nu) \leq C
$$

for all $(r, t, \nu) \in K \times K \times \mathbb{R}^{N}$ with $|\nu|=1$. 
From the lower semicontinuity of $\mathcal{F}_{\phi^{h}}(u)$, from (4.18) and (4.19), we then get that

$$
\begin{aligned}
& \liminf _{n \rightarrow+\infty} \int_{\Omega^{\prime} \cap J_{u_{n}}} \phi\left(x, u_{n}^{-}, u_{n}^{+}, \nu_{u_{n}}\right) d \mathcal{H}^{N-1} \\
\geq & \liminf _{n \rightarrow+\infty} \int_{\Omega^{\prime} \cap J_{u_{n}}} \phi^{h}\left(x, u_{n}^{-}, u_{n}^{+}, \nu_{u_{n}}\right) d \mathcal{H}^{N-1} \geq \int_{\Omega^{\prime} \cap J_{u}} \phi^{h}\left(x, u^{-}, u^{+}, \nu_{u}\right) d \mathcal{H}^{N-1} \\
\geq & \int_{\left(\Omega^{\prime} \backslash A_{h}\right) \cap J_{u}} \phi\left(x, u^{-}, u^{+}, \nu_{u}\right) d \mathcal{H}^{N-1}-\int_{\Omega^{\prime} \cap J_{u}} \widetilde{\varphi}_{h}(x) \psi_{h}\left(u^{-}, u^{+}, \nu_{u}\right) d \mathcal{H}^{N-1} \\
\geq & \int_{\left(\Omega^{\prime} \backslash A_{h}\right) \cap J_{u}} \phi\left(x, u^{-}, u^{+}, \nu_{u}\right) d \mathcal{H}^{N-1}-C \int_{\Omega^{\prime} \cap J_{u}} \widetilde{\varphi}_{h}(x) d \mathcal{H}^{N-1} .
\end{aligned}
$$

Since $\widetilde{\varphi}_{h} \rightarrow 0$ strongly in $W^{1,1}\left(\mathbb{R}^{N}\right)$ as $h \rightarrow \infty$, we have that, up to a subsequence, $\widetilde{\varphi}_{h}(x) \rightarrow 0$ for $\mathcal{H}^{N-1}$-almost every $x \in \mathbb{R}^{N}$ (see Proposition 1.2 in [16]). Therefore, by letting $h \rightarrow+\infty$ and recalling that $A_{h+1} \subset A_{h}$ for all $h$ and that $\mathcal{H}^{N-1}\left(\cap_{h} A_{h}\right)=0$, from the Dominated Convergence Theorem we get (4.6) in $\Omega^{\prime}$. Hence, by letting $\Omega^{\prime} \nearrow \Omega$, the thesis is achieved.

Acknowledgements. - I would like to thank G. Crasta and N. Fusco for some helpful discussions.

\section{REFERENCES}

[1] M. Amar, V. De Cicco, Relaxation in BV for a class of functionals without continuity assumptions. NoDEA Nonlinear Differential Equations Appl. (1-2)15 (2008) 25-44.

[2] M. Amar, V. De Cicco, Lower semicontinuity for polyconvex integrals without coercivity assumptions. Evol. Equ. Control Theory (2014).

[3] M. Amar, V. De Cicco, N. Fusco, A relaxation result in BV for integral functionals with discontinuous integrands. ESAIM Control Optim. Calc. Var. 13 (2007), no. 2, 396-412.

[4] M. Amar, V. De Cicco, N. Fusco, Lower semicontinuity and relaxation results in BV for integral functionals with BV integrands. ESAIM Control Optim. Calc. Var. 14 (2008), no. 3, 456-477.

[5] M. Amar, V. De Cicco, N. Fusco, Lower semicontinuity results for free discontinuity energies. Math. Models Methods Appl. Sci. 20 (2010), no. 5, 707-730.

[6] L. Ambrosio, A compactness theorem for a special class of function of bounded variation. Boll. Un. Mat. Ital. B(7) 3 (1989), 857-881. 
[7] L. Ambrosio, Existence theory for a new class of variational problems. Arch. Rat. Mech. Anal. 111 (1990) 291-322.

[8] L. Ambrosio, On the lower semicontinuity of quasi-convex integrals in SBV. Nonlinear Anal. 23 (1994), 405-425.

[9] L. Ambrosio, A new proof of the SBV compactness theorem. Calc. Var. Partial Differential Equations 3 (1995), 127-137.

[10] L. Ambrosio, A. Braides, Functionals defined on partitions in sets of finite perimeter I: integral representation and $\Gamma$-convergence. J. Math. Pures Appl. 69 (1990), 285306.

[11] L. Ambrosio, A. Braides, Functionals defined on partitions in sets of finite perimeter II: semicontinuity, relaxation, homogenization. J. Math. Pures Appl. 69 (1990), 307333.

[12] L. Ambrosio, G. Crasta, V. De Cicco, G. De Philippis, A nonautonomous chain rule formula in $W^{1, p}$ and in $B V$. Manuscripta Math. 140 (2013), no. 3-4, 461-480.

[13] L. Ambrosio, N. Fusco, D. Pallara, Functions of bounded variation and free discontinuity problems. Oxford Monographs, The Clarendon Press Oxford University Press, New York (2000).

[14] G. I. Barenblatt, The mathematical theory of equilibrium cracks in brittle fracture. Adv. Appl. Mech. 7 (1962), 55-129.

[15] M. Carriero, G. Dal Maso, A. Leaci, E. Pascali, Relaxation of the non-parametric Plateau problem with an obstacle. J. Math. Pures Appl. 67 (1988), 359-396.

[16] G. Dal Maso, On the integral representation of certain local functionals. Ricerche Mat. 32 (1983), 85-113.

[17] G. Dal Maso, A. DeSimone, M.G. Mora, Quasistatic evolution problems for linearly elastic-perfectly plastic materials. Arch. Ration. Mech. Anal. 180 (2006), 237-291.

[18] G. Dal Maso, G.A. Francfort, R. Toader, Quasistatic crack growth in nonlinear elasticity. Arch. Ration. Mech. Anal. 176 (2005), 165-225.

[19] G. Dal Maso, C. Sbordone, Weak lower semicontinuity of polyconvex integrals: a borderline case. Math. Z. 218 no. 4, (1995), 603-609.

[20] G. Dal Maso, R. Toader, A model for the quasistatic growth of brittle fractures, existence and approximation results. Arch. Ration. Mech. Anal. 162 (2002), 101135.

[21] G. Dal Maso, C. Zanini, Quasi-static crack growth for a cohesive zone model with prescribed crack path. Proc. Roy. Soc. Edinburgh Sect. A 137 (2007), 253-279.

[22] V. De Cicco, N. Fusco, A. Verde, On $L^{1}$-lower semicontinuity in $B V(\Omega)$. J. Convex Analysis 12 (2005), 173-185.

[23] V. De Cicco, N. Fusco, A. Verde, A chain rule formula in $\mathrm{BV}(\Omega)$ and its applications to lower semicontinuity. Calc. Var. Partial Differential Equations 28 (2007), 427447.

[24] V. De Cicco, G. Leoni, A chain rule in $L^{1}($ div; $\Omega$ ) and its applications to lower semicontinuity. Calc. Var. Partial Differential Equations 19 (2004), 23-51. 
[25] De Giorgi E. Semicontinuity theorems in the calculus of variations. With notes by U. Mosco, G. Troianiello and G. Vergara and a preface by C. Sbordone. Quaderni dell'Accademia Pontaniana, 56, (2008).

[26] H. Federer, W. P. Ziemer, The Lebesgue set of a function whose distribution derivatives are $p$-th power summable. Indiana Un. Math. J. 22 (1972), 139-158.

[27] I. Fonseca, G. Leoni, Modern Methods in the Calculus of Variations: $L^{p}$ Spaces. Springer Monographs in Mathematics, Springer (2007).

[28] G.A. Francfort, J.J. Marigo, Revisiting brittle fracture as an energy minimization problem. J. Mech. Phys. Solids 46 (1998), 1319-1342.

[29] N. Fusco, M. Gori, F. Maggi, A remark on Serrin's Theorem. NoDEA Nonlinear Differential Equations Appl. 13 (2006), 425-433.

[30] G. Lazzaroni, Quasistatic crack growth in finite elasticity with Lipschitz data. Ann. Mat. Pura Appl. 190 (2011), 165-194.

Dipartimento S.B.A.I., Scienze di Base e Applicate per l'Ingegneria, UniverSità di Roma "La Sapienza", Via A. Scarpa 16, 00161 Roma, Italy

E-mail address: virginia.decicco@sbai.uniroma1.it 